Note

\section{Solvent Effects on Circular Dichroism of Colominic Acid}

\author{
Kunihiko GEKKo \\ Department of Food Science and Technology, \\ Faculty of Agriculture, Nagoya University, \\ Nagoya 464, Japan
}

Received August 23, 1979

The conformation of colominic acid, a homopolymer of $N$-acetyl-neuraminic acid, has been investigated through the effects of solvents on the optical rotation." Although the circular dichroism (CD) spectra of this polymer have been recorded by two groups, ${ }^{2.3}$ the solvent effects on the spectra are not clarified. In this work, the effects of $\mathrm{pH}$, urea and guanidine hydrochloride on the CD spectra of this polymer were examined to obtain supplementary information on its conformation in water. Sodium salt of colominic acid containing over $95 \% \mathrm{~N}$-acetylneuraminic acid from $E$. Coli 016:K1: HNM was purchased from Nakarai Chemicals, Ltd. (lot No. V6G 5478, $M w=10^{4}$ ). After dialysis against distilled water at $4{ }^{\circ} \mathrm{C}$, this polymer solution was passed through a cation-exchange resin column (Amberlite IR-120) to change completely the polymer into a sodium or acidic form, and then it was lyophilized. The crystalline $\mathrm{N}$-acetylneuraminic acid (sialic acid) containing over $99.0 \%$ purity (lot No. M7M1318) was obtained from Nakarai Chemicals, Ltd. The ultra-pure urea was obtained from Schwarz/Mann. Guanidine hydrochloride $(\mathrm{GuHCl})$ was a special grade reagent of over $99.5 \%$ purity from Nakarai Chemicals, Ltd. Circular dichroism was measured at $25^{\circ} \mathrm{C}$ with a Jasco J-40 spectropolarimeter which was calibrated with d-10-camphor sulfonic acid in water. The results of $\mathrm{CD}$ are expressed in terms of molar ellipticity, $[\theta]$, in $\mathrm{deg} \cdot \mathrm{cm}^{2} / \mathrm{dmol}$ without correction of the Lorenz refractive index factor, by using the mean residue molecular weights of 291 and 313 for the monomeric units of colominic acid and its sodium salt, respectively. The molecular weights of sialic acid and its sodium salt were 309 and 331 , respectively.

Prior to elucidating the effect of $\mathrm{pH}$, the time course of $\mathrm{CD}$ spectrum of this polymer in the acid region was examined. The ellipticity of negative band increased, accompanying the shift of the band from $228 \mathrm{~nm}$ (sodium form) to $235 \mathrm{~nm}$ (acid form), with the lapse of time after the $\mathrm{pH}$ was adjusted to 2.0 with $\mathrm{HCl}$. This conversion of spectrum was accelerated at higher temperature, and the ellipticity leveled off about one day after the preparation. When thus prepared acid solution was again neutralized with $\mathrm{NaOH}$, the spectrum recovered very slowly. These time-dependences are similar to those in the $\mathrm{pH}$ titration experiment reported by McGuire and Binkley. ${ }^{4}$ However, no time-dependence was observed in the specific viscosity of the solution at $\mathrm{pH} 2.0$. This indicates that the observed time-dependence in $\mathrm{CD}$ spectra is due to a local configurational change of chromophore groups which does not influence the gross conformation of the polymer.

Figure 1 shows the $C D$ spectra of colominic acid sodium salt at various $\mathrm{pH}$ values adjusted with $\mathrm{HCl}$ or $\mathrm{NaOH}$, the polymer concentration being fixed at $0.05 \%$. The $C D$ spectrum of colominic acid ( $\mathrm{pH} 3.6$ ) was characterized by a negative band at $234 \mathrm{~nm}([\theta]=-9,000)$ and a positive band at $197 \mathrm{~nm}([\theta]=27,800)$ with a crossover at $207 \mathrm{~nm}$. This result is very close to the previously reported one for the B polysaccharide. ${ }^{3)}$ With an increase in $\mathrm{pH}$ value, the positive band and the crossover point shifted to longer wavelengths and the negative band, to shorter wavelengths, accompanied by a significant decrease in ellipticity. Since the spectrum of sialic acid shows a similar, though not so significant, $\mathrm{pH}$-dependence, the assignment for the $\mathrm{CD}$ spectrum of sialic acid ${ }^{5 /}$ may be applied to the positive band of the polymer. If this is the case, the positive band of colominic acid would be attributed to the mixture of carboxyl $\mathrm{n}-\pi^{*}$ and amide $\mathrm{n}-\pi^{*}$ and $\pi-\pi^{*}$ transitions.

Kabat et al. ${ }^{2)}$ assumed that the origin of the negative band was the acetamide groups inaccessible to solvent,

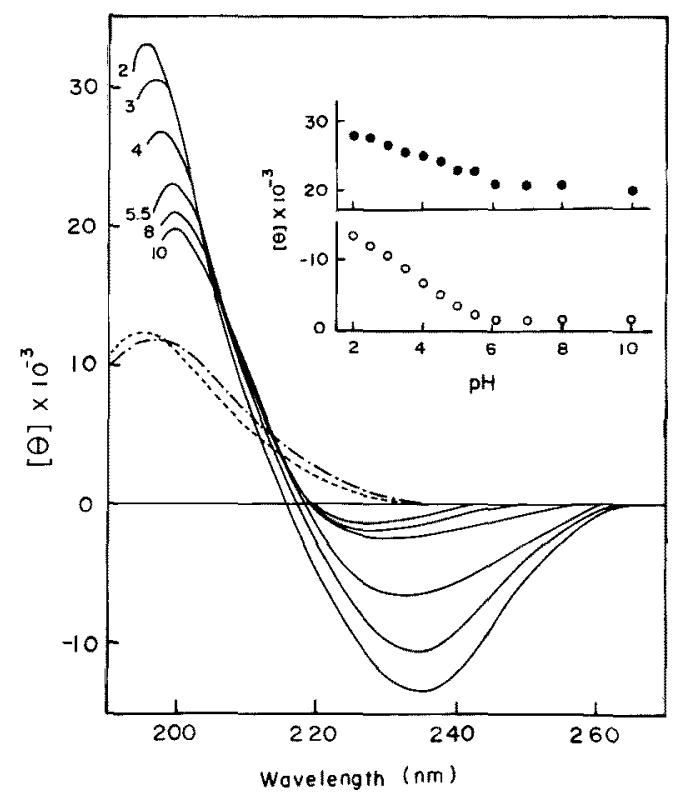

Fig. 1. Effect of $\mathrm{pH}$ on CD Spectra of Colominic Acid Sodium Salt.

The number attached to the curve shows the $\mathrm{pH}$ value of solution. The dotted and broken lines show the data for sialic acid and its sodium salt, respectively. The values of [ध] at $200 \mathrm{~nm}(0)$ and $235 \mathrm{~nm}(O)$ are plotted as a function of $\mathrm{pH}$ in the inset. 
brought about by the folded polymer conformation. However, Hirano and Kondo ${ }^{13}$ found that the specific rotation of colominic acid was not affected by $8 \mathrm{M}$ urea and this polymer had a random coil conformation in a single strand. Certainly, the viscosity behavior of this polymer was characteristic of the flexible polyelectrolyte chain: the specific viscosity of sodium form polymer in water at $25^{\circ} \mathrm{C}$ increased significantly with a decrease in polymer concentration (the specific viscosities were $0.21,0.16,0.14$ and $0.11 \mathrm{dl} / \mathrm{g}$ at the polymer concentrations of $0.04,0.10,0.15$ and $0.30 \mathrm{~g} / \mathrm{dl}$, respectively), but such concentrationdependence dissapeared upon addition of salt, and the intrinsic viscosity decreased to 0.06 and $0.04 \mathrm{dl} / \mathrm{g}$ in 0.01 and $0.1 \mathrm{M} \mathrm{NaCl}$, respectively. Although the negative band should be influenced by dilution if an expansion of polymer chain caused further exposure of the acetamide groups to solvent, the magnitude and position of negative band was independent of the polymer concentration in the concentration range of 0.01 to $0.30 \mathrm{~g} / \mathrm{dl}$. Furthermore, a recent NMR study $y^{6}$ revealed that the environment around acetamide group of polymer was not different from that of monomer. In view of these results, it is improbable that the polymer is unfolded in such a way as to involve the acetamide groups newly exposed to the solvent. Recently, Jennings and Williams ${ }^{3)}$ proposed that the carboxyl group could be involved in the generation of the negative band either directly through the forbidden carboxyl $n-\pi *$ transition or through perturbation of the $n-\pi^{*}$ transition of the amide chromophore by the carboxyl group. While such perturbation would be possible through the hydrogen bond between carboxyl group and acetamide group, there is no evidence for such a hydrogen bond in NMR data. ${ }^{6 /}$ Thus, two bands with different signs are expected to arise mainly from the $n-\pi^{*}$ transition of different rotational isomers of carboxyl chromophore as have been observed for some sugars or polysaccharides involving carboxyl groups. "A part of the origin of such rotational isomers, being not well known, would be an electronic perturbation and a hydrogen bonding ability of carboxyl group. "In this connection $_{*}$ it is profitable to know the effects of denaturants and temperature on the negative band. As shown in Fig. 2, both urea and $\mathrm{GuHCl}$ gradually depressed the generation of the negative band with an increase in their concentrations. This result, too, must reflect the local configurational change of carboxyl chromophore since the gross conformation is not affected by $8 \mathrm{M}$ urea. ${ }^{\prime \prime} \mathrm{A}$ temperature increase by $10 \mathrm{C}$ brought about an approximately $5 \%$ decrease in the magnitude of negative band in the temperature range of 18 to $51^{\circ} \mathrm{C}$. Although these results suggest the existence of hydrogen-bonded carboxyl group contributing to the negative band, it cannot be determined from the findings of the present work whether an intramolecular lactone structure or ester linkage proposed ${ }^{4}$ exists or not

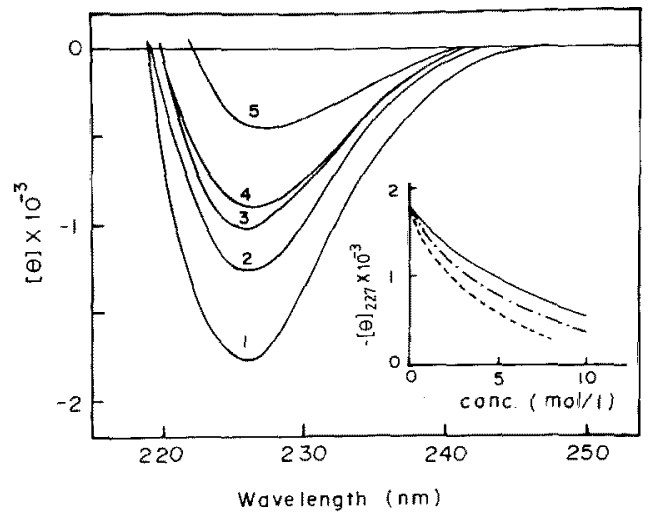

F1G. 2. Effect of Urea and $\mathrm{GuHCl}$ on CD Spectra of Colominic Acid Sodium Salt in Phosphate Buffer (pH 7.0, ionic strength 0.1 ).

Curve 1, buffer only; curve 2, $3 \mathrm{M}$ urea; curve $3,4 \mathrm{M}$ GuHCl; curve 4, $6 \mathrm{M}$ urea; curve 5, $6 \mathrm{M} \mathrm{GuHCl}$. The inset is the plot of $[\theta]$ at $227 \mathrm{~nm}$ as a function of the concentrations of urea $\left(-, 25^{\circ} \mathrm{C}\right.$;.,$\left.-- 45^{\circ} \mathrm{C}\right)$ and $\mathrm{GuHCl}\left(---, 25^{\circ} \mathrm{C}\right)$.

since the characteristic band based on such structure overlaps the carboxyl $n-\pi^{*}$ transition. However, this does not necessarily exclude the possibility of such a structure formation, as expected from the time-dependence of the acid CD spectrum. It is interesting to note that, in NMR of colominic acid, ${ }^{6}$ only $\mathrm{C}(9)$ resonance is different from all other carbon atoms, which moves upfield as compared with that of sialic acid, in accordance with the expectation of an esterification of the 9 -position. ${ }^{4}$

\section{REFERENCES}

1) S. Hirano and S. Kondo-Ikeda, Biopolymers, 13, 1357 (1974).

2) E. A. Kabat, K. O. Lloyd and S. Beychok, Biochemistry, 8, 747 (1969).

3) H. J. Jennings and R. E. Williams, Carbohydr, Res., 50, 257 (1976)

4) E. J. McGuire and S. B. Binkley, Biochemistry, 3, 247 (1964)

5) H. R. Dickinson and C. A. Buck, Biochemistry, 14, 2299 (1975).

6) A. K. Bhattacharjee, H. J. Jennings, C. P. Kenney, A. Martin and I. C. P. Smith, J. Biol. Chem., 250, 1926 (1975).

7) K. Gekko, Biopolymers, 18, 1989 (1979). 